\title{
Observing Extrasolar Planetary \\ Systems with ALMA
}

\author{
Bryan Butler, Alwyn Wootten, Antonio Hales \& Bob Brown ${ }^{1}$ \\ National Radio Astronomy Observatory
}

\begin{abstract}
We address in this white paper the ability of ALMA to observe extrasolar planetary systems (in various stages of formation). The observation of extrasolar planetary systems is thought to be one of the most important science drivers for ALMA. As such, we should have some idea of what the capabilities might be in this regard.
\end{abstract}

\section{Introduction}

Recently, much emphasis has been placed on the optical and infrared wavelengths to observe extrasolar planets and planetary systems. We suggest here that the millimeter $(\mathrm{mm})$ and submillimeter (sub-mm) regions of the spectrum offer an attractive alternative to those shorter wavelengths. ALMA will be able to detect planetary systems at all evolutionary stages. In this paper, we concentrate in the abilities that ALMA will have for observing young, forming giant planets ("condensations") in protoplanetary disks (and the gaps created in these disks as the condensations grow), as well as for detecting giant planets around nearby stars, either through direct imaging or indirect detection through the use of high-precision astrometry. For a more in-depth discussion on ALMA capabilities for observing circumstellar disks at different stellar ages, please refer to ALMA Memo $475^{2}$ (Butler, Wootten \& Brown 1999).

\section{The Instrument}

The Atacama Large Millimeter/submillimeter Array (ALMA), will be a single research instrument composed of up to 80 high-precision antennas, located on the Chajnantor plain of the Chilean Andes in the District of San Pedro de Atacama, $5000 \mathrm{~m}$ above sea level. These antennas will have superb surface accuracy - better than $20 \mu \mathrm{m}$, and extremely good pointing - better than 0.6 arcseconds. The frequency coverage of ALMA will include receivers in all of the atmospheric windows from 31 to $950 \mathrm{GHz}$ ( $7 \mathrm{~mm}$ to $350 \mu \mathrm{m})$. These receivers will be state of the art, with extremely good noise characteristics (as good as 3 times quantum limited). Total processed bandwidth will be $16 \mathrm{GHz}$ per baseline in continuum mode. The spectral resolution is expected to be better than

\footnotetext{
${ }^{1}$ now at National Astronomy and Ionosphere Center

${ }^{2}$ http: / /www.alma.nrao.edu/memos/html-memos/abstracts/abs 475 . html
} 
Table 1: Sensitivity for ALMA in 1 minute.

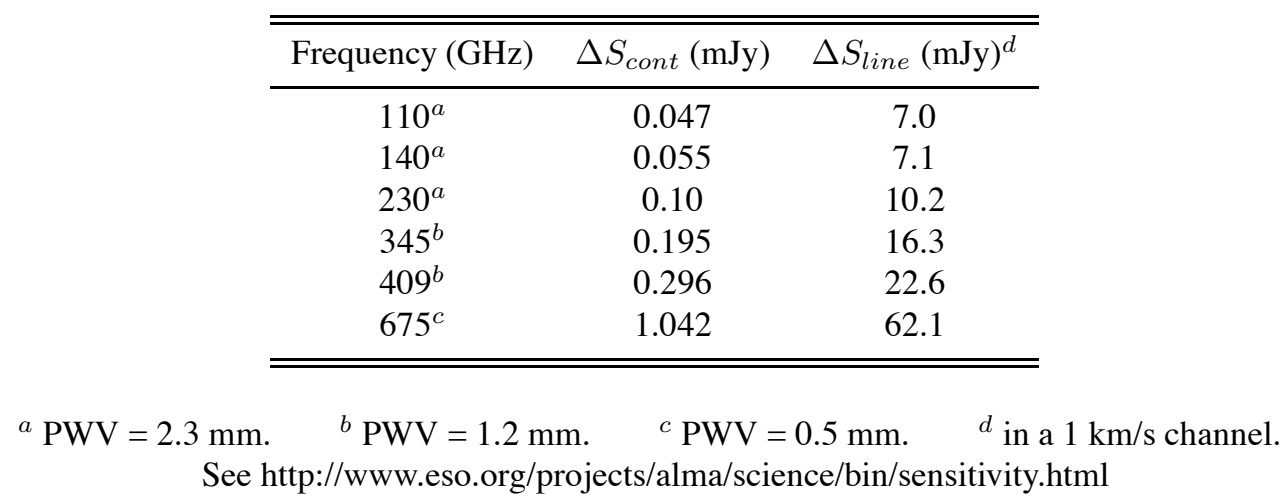

$0.05 \mathrm{~km} / \mathrm{s}$. Full polarization information will be available on all baselines. The antennas will be arranged in a number of different configurations, with the widest having maximum baselines of $\sim$ $15 \mathrm{~km}$ - providing imaging with resolution finer than 10 milliarcseconds. The expected sensitivity for ALMA in both continuum and spectral line modes is shown in Table 1. This is the sensitivity with only 1 minute of integration (Butler \& Wootten 1999).

\section{Direct Detection}

The possibility of direct detection of planets around other stars is an exciting one, and we explore that possibility here. By direct detection, we mean the direct measurement of the emission (thermal or otherwise) from a "planet" and its central star, and hence the ability to produce an image of the star and the planet, albeit with only 1 pixel on each object. For the purposes of this section, "planet" means a distinct body orbiting a central star. The only such bodies luminous enough to detect are gaseous giant planets. However, they may be at any stage of their evolution, i.e., they may be very young, and hence very large and hot, or they may be quite mature (like Jupiter). The conditions necessary for such a detection are:

1. there must be sufficient flux density from the planet to obtain a "reasonable" signal to noise ratio (SNR), in a "reasonable" amount of time.

2. the emission from the planet must be distinguished from that from the star.

3. the detection must be obtained in a short enough time that the planet does not move too far in the plane of the sky.

\subsection{Calculating Detectability}

Here we consider the flux density from the planet compared to the expected noise characteristics of ALMA. Assuming that we are in the Rayleigh-Jeans regime, the flux density from the planet at 
a wavelength $\lambda$ is given by:

$$
F_{\lambda} \approx \frac{2 k T}{\lambda^{2}} \frac{\pi R^{2}}{D^{2}}
$$

where $R$ is the radius of the effective emitting region of the planet, $D$ is the distance to the system, $k$ is the Boltzmann constant, and $T$ is the equivalent temperature of the planet. For observations of any thermal blackbody (with emission which goes like $\lambda^{-2}$ ), and given the ALMA sensitivities listed in Table 1, the SNR of a detection is maximized at $345 \mathrm{GHz}$ (since $\mathrm{SNR} \propto \frac{\nu^{2}}{\Delta S}$, where $\Delta S$ is the noise at frequency $\nu$.)

As examples, we will consider 3 different types of giant planets, corresponding to different evolutionary ages. First, a mature giant planet similar to our own Jupiter: $R \sim 7.0 \times 10^{7} \mathrm{~m} \sim 1 R_{j}$; $T \sim 200 \mathrm{~K}$. Second, a mature, but hotter planet (which might be considered a brown dwarf, e.g., G1229B): $R \sim 1.0 \times 10^{8} \mathrm{~m} \sim 1.5 R_{j} ; T \sim 1000 \mathrm{~K}$. And lastly, a very young "protoJupiter": $R \sim 2.1 \times 10^{9} \mathrm{~m} \sim 30 R_{j} ; T \sim 2500 \mathrm{~K}$. Values of the flux density for these objects at $1,5.7,10$ and 120 parsecs are shown in Table 2, for a frequency of $345 \mathrm{GHz}$.

Table 2: Giant planet flux densities at $345 \mathrm{GHz}$ (in $\mu \mathrm{Jy}$ ).

\begin{tabular}{cccc}
\hline \hline distance (pc) & Case 1 (Jupiter) & Case 2 (G1229B) & Case 3 (protoJupiter) \\
\hline 1 & 12 & 130 & 59000 \\
5.7 & 0.36 & 4.1 & 1820 \\
10 & 0.12 & 1.3 & 590 \\
120 & 0.0008 & 0.009 & 4.1 \\
\hline \hline
\end{tabular}

Now, are these levels of flux density detectable with ALMA? We take the noise flux density at $345 \mathrm{GHz}$ as $0.11 \mathrm{mJy}$ in 1 minute (Butler \& Wootten 1999). Given this, and presuming that we want to make these detections with a reasonable SNR (we choose 5), the times required for detections of the flux density levels shown in Table 2 are shown in Table 3. It can be clearly seen that the detection of any mature giant planet (like Jupiter) would only be feasible for the very nearest stars. However, the direct detection of the very young, hot, protoJupiters in the nearest star forming regions is very feasible, given the sensitivity of ALMA.

The separation of the protoJupiter from the central protostar must be sufficiently large to allow for discrimination between the two. For a face-on system, this separation is simply: $\theta_{\text {sep }}=a / D$. This may force us to carry out the observations in the largest possible configuration of ALMA, as for observations at $345 \mathrm{GHz}$ with maximum baselines of $4 \mathrm{~km}$, the resolution of ALMA will be $\sim 45$ milliarcseconds. For a system at $120 \mathrm{pc}$ with a protoJupiter at $5 \mathrm{AU}$ orbital radius, $\theta_{\text {sep }} \sim 40$ milliarcseconds. So, we may have to utilize the $15 \mathrm{~km}$ configuration, where the resolution is $\sim 12$ milliarcseconds.

In addition, the integration times shown in Table 3 should be short enough that the protoJupiter does not move in its orbit so much as to move through a significant portion of a resolution element. 
Table 3: Integration time (days) needed to detect extrasolar planets.

\begin{tabular}{cccc}
\hline \hline distance (pc) & Case 1 (Jupiter) & Case 2 (G1229B) & Case 3 (protoJupiter) \\
\hline 1 & 1.5 & 0.01 & + \\
5.7 & $*$ & 12.5 & + \\
10 & $*$ & 120 & + \\
120 & $*$ & $*$ & 12.5 \\
\hline \hline
\end{tabular}

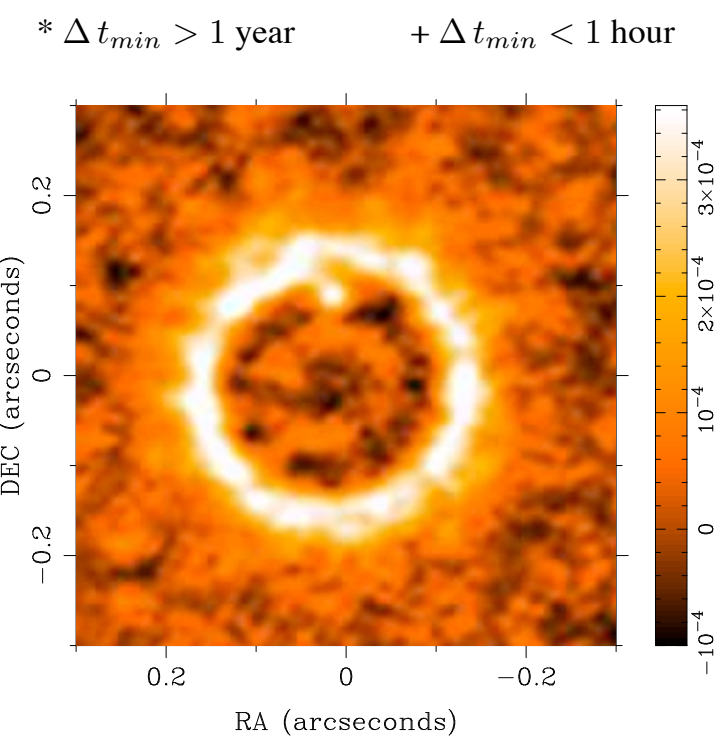

Figure 1: ALMA $345 \mathrm{GHz}$ simulated observations of a protoJupiter embedded in a $0.02 M_{\odot}$ protoplanetary disk located $50 \mathrm{pc}$ away from the Sun. The orbital radius is $5 \mathrm{AU}$, and the mass of the central star (not included in this image) is $0.5 M_{\odot}$. Original model image, courtesy S. Wolf (from Wold \& D'Angelo 2005

If we demand that the protoJupiter move less than $1 / 4$ of a resolution element, for observations at $345 \mathrm{GHz}$ and maximum baselines of $15 \mathrm{~km}$, the maximum observation time is $\sim 8$ days for a protoJupiter at $5 \mathrm{AU}$ orbital radius around a solar mass central protostar, at $120 \mathrm{pc}$. So, this constraint should not be a problem. Even in the case where the motion begins to be an appreciable fraction of a resolution element, one can attempt to remove the motion (by searching the phase space of possible masses and orbits) in the interferometric visibilities, minimizing the effect.

The above treatment has ignored the flux density from the central protostar. At optical and infrared wavelengths, the confusion from the central protostar may be a problem, as it can be as much as 6 orders of magnitude brighter than the protoJupiter. Fortunately, this ratio is much less at sub-mm and mm wavelengths. Typical ratios at these wavelengths should be only on the order of 1000 or so, posing no dynamic range problem for ALMA. In fact, the flux density from the central protostar is an aid in imaging with ALMA, as it could be used to maintain the coherence of the 
instrument (provided its flux density is large enough).

\section{Indirect Detection (Astrometry)}

The orbit of any planet around its central star causes that star to undergo a reflexive circular motion around the star-planet barycenter. By taking advantage of the incredibly high resolution of ALMA in its widest configuration, we may be able to detect this motion. Making the usual approximation that the planet mass is small compared to the stellar mass, the stellar orbit projected on the sky is an ellipse with angular semi-major axis $\theta_{r}$ (in $\operatorname{arcsec}$ ) given by:

$$
\theta_{r}=\frac{m_{p}}{M_{*}} \frac{a_{A U}}{D_{p c}}
$$

where $m_{p}$ is the mass of the planet, $M_{*}$ is the mass of the star, $a_{A U}$ is the orbital distance of the planet (in $\mathrm{AU}$ ), and $D_{p c}$ is the distance to the system (in parsecs).

The astrometric resolution of ALMA, or the angular scale over which changes can be discriminated $(\Phi)$, is proportional to the intrinsic resolution of ALMA, and inversely proportional to the signal to noise with which the stellar flux density is detected $\left(\mathrm{SNR}_{*}\right)$ :

$$
\Phi=\frac{\theta_{H P B W}}{2 \cdot \mathrm{SNR}_{*}}
$$

This relationship provides the key to high precision astrometry: the astrometric accuracy increases both as the intrinsic resolution improves and also as the signal to noise ratio is increased. Astrometry at radio wavelengths routinely achieves absolute astrometric resolutions 100 times finer than the intrinsic resolution, and can achieve up to 1000 times the intrinsic resolution with special care. The phase stability specifications for ALMA will allow such astrometric accuracy to be achieved for wide angle astrometry.

When the astrometric resolution is less than the reflexive orbital motion, that is, when $\Phi \lesssim \theta_{r}$, ALMA will detect that motion. As above, we use the approximation that $\theta_{H P B W} \sim \lambda / B_{\max }$, so that detection will occur when:

$$
\mathrm{SNR}_{*} \gtrsim 10^{5} \frac{\lambda}{B_{\max }}\left(\frac{m_{p}}{M_{*}} \frac{a_{A U}}{D_{p c}}\right)^{-1} .
$$

The factor of $2 \times 10^{5}$ enters in to convert from radians to arcseconds.

Plugging numbers into the above equation for the giant planets in our own solar system shows that ALMA will detect the reflex motion of systems with such planets as far away as $\mathrm{SNR}_{*}$ parsecs distant (i.e., the ratio of $\mathrm{SNR}_{*}$ to $D_{p c}$ is roughly 1 for our own giant planets). So, e.g., if we can reach an SNR of 10 on the central star, we can detect companions around such stars to 10 parsecs. If we use the expected thermal flux density of the Sun at $345 \mathrm{GHz}$ as a guide, the received flux density from other similar stars would be:

$$
F_{*} \sim \frac{30}{D_{p c}^{2}} \mathrm{mJy}
$$


Given that the noise of ALMA at $345 \mathrm{GHz}$ will be about $0.1 \mathrm{mJy} / \mathrm{min}$, an $\mathrm{SNR}_{*}$ of 10 should be achieved in about $10 \mathrm{~min}$ for a star at 10 parsecs distance. Note, however, that astrometric detection of a planet requires that curvature in the apparent stellar motion be measured, since linear terms in the reflex motion are indistinguishable from ordinary stellar proper motion. This implies that at the very minimum, one needs three observations spaced in time over roughly half of the orbital period of the observed system. A detection of a planetary system with astrometry would thus require some type of periodic monitoring.

\subsection{Number of Stars with Detectable Wobble}

If all of the detectable stars had planetary companions, how many of them could be detected (via astrometry) with ALMA? We address that question in this section. We reject all variable and multiple star systems from consideration. This is rather strict, but we prefer to avoid the complications of these systems for astrometry and planet detection.

We assume that the planets are in orbits with semimajor axis of $5 \mathrm{AU}$. We consider 3 masses of planetary companions: 5 times Jovian, Jovian, and Neptunian. We assume integration times of 10 minutes, again at $345 \mathrm{GHz}$. From the Hipparcos catalog (Perryman et al.1997), there are $\sim 800$ stars around which a $5^{*}$ Jovian companion could be detected, $\sim 180$ stars around which a Jovian companion could be detected, and no stars around which a Neptunian companion could be detected. Again, virtually none of these stars are solar-type. From the Gliese catalog (Gliese $\&$ Jahreiss 1988), there are $\sim 200$ stars around which a $5^{*}$ Jovian companion could be detected, $\sim 120$ stars around which a Jovian companion could be detected, and $\sim 30$ stars around which a Neptunian companion could be detected. Of these, close to 100 of the $5 *$ Jovians are solar-type, close to 30 of the Jovians are solar-type, and none of the Neptunians are solar-type.

\subsection{Comparisons with Other Techniques}

Ground based efforts to find extra solar planets have focused in recent years in several areas, including differential astrometry, radial velocity measurements, and gravitational lensing. There are also proposals for methods which could directly image Jovian-class planets near a small number of stars. Planet detection using astrometry with ALMA will both complement these efforts, and have several important advantages:

- Astrometric searches for planets with ALMA will use absolute (wide-angle) astrometry, avoiding the problem of solving for motions in both a target star and a background reference star. ALMA will be able to tie stellar positions directly to the quasar reference frame, since both stars and quasars will be bright enough for high precision astrometry with ALMA.

- Systematic errors will be lower with ALMA than with techniques at optical or IR wavelengths because ALMA can observe stars 24 hours a day, providing various types of closure 

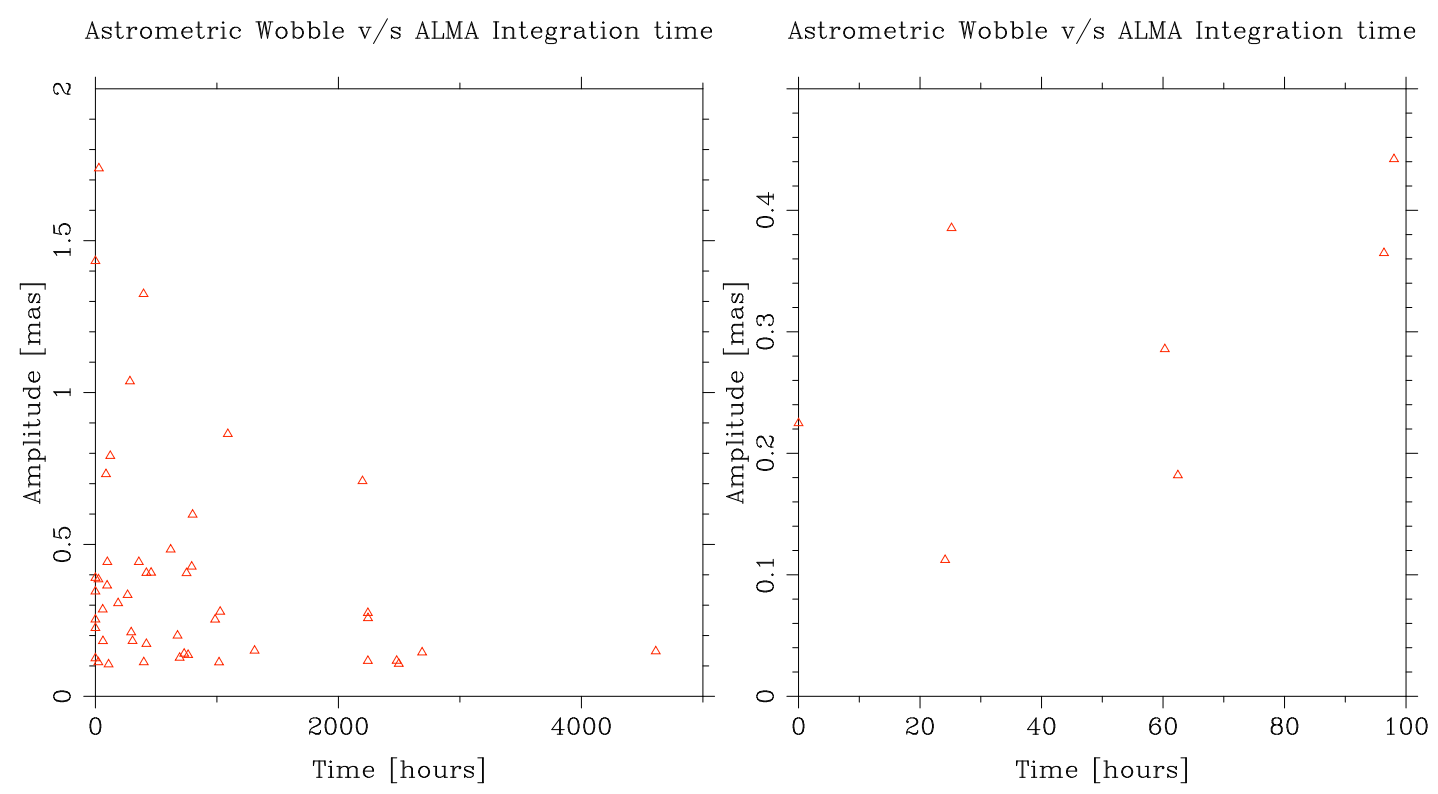

Figure 2: Left-hand panel: Amplitude of the astrometric wobble of known exoplanet host stars versus the integration time required in order to achieve the predicted astrometrical precision of 0.1 milliarcseconds. System characteristics from the California and Carnegie Planet Search (http://exoplanets.org). Right-hand panel: Blown-up of the region probable with ALMA.

constraints on ALMA astrometry. This will reduce the kinds of seasonal systematic errors that plague optical astrometry.

- Astrometry measures the position of the star, so that the mass of the unseen planet can be easily determined. Direct detection techniques, either ground or space-based, cannot determine planetary masses, but will provide constraints on the parameters of a particular planetary system that complement those provided by astrometry.

- Astrometric results from ALMA will directly complement the results expected from differential astrometry with proposed ground-based IR interferometers. ALMA will not face the constraints on finding suitable background reference stars that are required by differential IR astrometry, so some additional stellar systems may be accessible to ALMA.

- As with other astrometric techniques, the detectability of a planet does not depend on the inclination of the planet's orbital plane around its primary star. In fact, astrometry could resolve inclination ambiguities for planets discovered using radial velocity techniques, if the amplitude of the astrometric signal is large enough.

- Astrometric searches are complementary to radial velocity searches in that the former are more sensitive to planets with larger semimajor axes, and the latter are more sensitive to 
ones with smaller semimajor axes.

\section{Summary}

ALMA will make substantial contributions to the observations of extrasolar planetary systems at all evolutionary stages. In particular, there are four areas in which ALMA will be able to aid in the investigation of extrasolar planets and planetary systems:

- ALMA will be able to directly detect forming giant planets ("condensations") in protoplanetary disks, and the gaps created in these disks as the condensations grow.

- ALMA will be able to directly detect very young giant planets in the nearest star forming regions.

- ALMA will be able to indirectly detect the presence of giant planets around nearby stars through the use of astrometry.

These observations are absolutely crucial to our understanding of how our own solar system formed, and may help in answering the question of whether we are alone in the universe.

\section{References}

Butler, B., \& A. Wootten, ALMA Sensitivity, Supra-THz Windows, and $20 \mathrm{~km}$ baselines, ALMA memo 276, 1999

Gliese, W, \& H. Jahreiss, The Third Catalogue of Nearby Stars. I. General View and Content, in Star Catalogues: a Centennial Tribute to A.N. Vyssotsky. Proc. Meeting IAU Comm. 24, Ed. A.G.D. Philip \& A.R. Upgren, L. Davis Press, 1988

Penny, A.J., P. Artymowicz, A.-M. Lagrange, \& S.S. Russell, Planetary Systems in the Universe: Observation, Formation, and Evolution, Proceedings of IAU Symposium 202, ASP Conference Series

Perryman M.A.C., \& 19 others, The Hipparcos Catalogue, A\&A, 323, L49-52, 1997

Wootten, A., Mangum, J. G., \& Holdaway, M, Debris Disks and the Formation of Planets, . ASP Conf. Ser. 324, 277 